\title{
The comparative effects of nutritional drinks designed to augment athletic performance and recovery
}

\author{
Douglas Kalman ${ }^{1 *}$, Samantha Feldman ${ }^{1}$, Elaine Morales ${ }^{1}$, Amy G Preston², Debra L Miller², Stephen Crozier², \\ Diane R Krieger ${ }^{1}$
}

From International Society of Sports Nutrition; 7th Annual ISSN Conference and Expo

Clearwater Beach, FL, USA. 24-26 June 2010

\section{Background}

The purpose of this study was to determine and compare the effects of 2 cocoa-based CHO-PRO beverages (3.5\% and 6\% natural cocoa) with a leading sports beverage [CHO-electrolyte solution (CES)] and placebo (CHO-PRO without cocoa) on exercise performance and recovery in healthy adult physically active males.

\section{Methods}

22 males $(24.9 \pm 4.4)$ completed 4 exercise test visits, each involving an exhaustive exercise protocol intended to induce muscle soreness (30 minutes, -10 degree decline, 75\% HRmax) and 4 hours later, a TTE performance trial. In a crossover, partially double-blinded manner, subjects were provided 2 servings of the beverage (11-13.7 oz), 15 minutes and 2 hours after the exhaustive exercise. Muscle recovery was assessed via the rate of return to baseline of CPK and LDH over the 72-hour post exercise period. Exercise test visits were at least 1 week apart to allow for muscle recovery.

\section{Results}

The TTE times for the $3.5 \%$ cocoa beverage were significantly longer than the times for placebo and CES; ( 85 seconds; $\mathrm{p}=0.042$ and 133 seconds; $\mathrm{p}=0.002$ respectively) and the times for the $6 \%$ cocoa beverage were significantly longer than the times for CES (114 seconds; $\mathrm{p}=0.009$ ) with no performance difference between the $3.5 \%$ and $6 \%$ cocoa beverages. In relative terms, the $3.5 \%$ cocoa beverage produced a $4.4 \%$ greater median increase in TTE versus placebo $(\mathrm{p}=0.039)$ and $11.3 \%$ increase versus $C E S$

Miami Research Associates. Endocrinology-Nutrition Division. 6141 Sunset Drive \#301 Miami, FL 33143, USA

Full list of author information is available at the end of the article $(\mathrm{p}=0.017)$ and the $6 \%$ cocoa beverage produced a $3.8 \%$ increase versus placebo $(\mathrm{p}=0.032)$ and $5.5 \%$ increase versus CES $(p=0.026)$. All 4 beverages had a significant return to baseline of CPK and LDH. The 3.5\% cocoa beverage showed a larger effect for LDH (85\% return versus $78 \%$ return to baseline for the other 3 beverages) and the 3.5\% cocoa beverage and placebo showed a larger effect for CPK as compared to the CES and 6\% cocoa beverage although these differences were not statistically significant.

\section{Conclusion}

The addition of cocoa to CHO-PRO beverages may offer an exercise performance advantage over CHO-PRO beverages without cocoa and $\mathrm{CHO}$-electrolyte solutions. This clinical trial found that a 3.5\% cocoa CHO-PRO beverage demonstrated significant performance enhancement effects as compared to placebo and a leading sports beverage. Additional studies are warranted to fully explore the potential ergogenic effects of the $3.5 \%$ cocoa beverage.

\section{Acknowledgement}

Miami Research Associates received study funding from The Hershey Company for this clinical trial. The authors would like to thank The Hershey Center for Health and Nutrition, The Hershey Company.

\section{Author details}

'Miami Research Associates. Endocrinology-Nutrition Division. 6141 Sunset Drive \#301 Miami, FL 33143, USA. ${ }^{2}$ The Hershey Center for Health and Nutrition, The Hershey Company, 1025 Reese Avenue, Hershey, PA 17033, USA.

Published: 15 September 2010

\section{doi:10.1186/1550-2783-7-S1-P15}

Cite this article as: Kalman et al:: The comparative effects of nutritional

drinks designed to augment athletic performance and recovery. Journal of the International Society of Sports Nutrition 2010 7(Suppl 1):P15. 